\section{(2) \\ BRAZIULIAN JOURNAL \\ OF MEDICAL AND BIOLOGICAL RESEARCH}

www.bjournal.com.br
ISSN 0100-879X

Volume 45 (6) 473-564 June 2012

BIOMEDICAL SCIENCES

AND

CLINICAL INVESTIGATION

Braz J Med Biol Res, May 2012, Volume 45(6) 516-523

doi: $10.1590 / \mathrm{S} 0100-879 X 2012007500046$

Longitudinal brain volumetric changes during one year in nonelderly healthy adults: a voxel-based morphometry study

R.M. Guimarães, M.S. Schaufelberger, L.C. Santos, F.L.S. Duran, P.R. Menezes, M. Scazufca, M.T.V Gouvea and G.F. Busatto

The Brazilian Journal of Medical and Biological Research is partially financed by

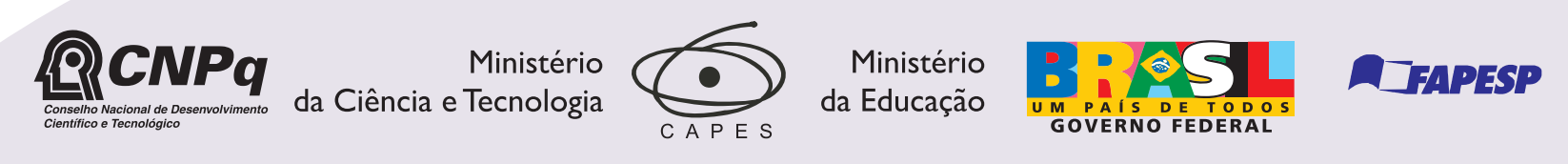

Institutional Sponsors

Scied
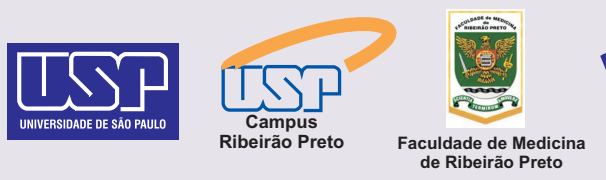

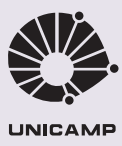

SHIMADZu

Associaçäo de Incentivo
à Pesquisa

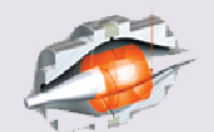




\title{
Longitudinal brain volumetric changes during one year in non-elderly healthy adults: a voxel-based morphometry study
}

\author{
R.M. Guimarães ${ }^{1}$, M.S. Schaufelberger ${ }^{1,2}$, L.C. Santos ${ }^{1}$, F.L.S. Duran ${ }^{1}$, P.R. Menezes ${ }^{3}$, \\ M. Scazufca ${ }^{1}$, M.T.V Gouvea ${ }^{1}$ and G.F. Busatto ${ }^{1}$ \\ ${ }^{1}$ Laboratório de Neuroimagem (LIM-21), Departamento de Psiquiatria, Faculdade de Medicina, \\ Universidade de São Paulo, São Paulo, SP, Brasil \\ ${ }^{2}$ Departamento de Neurociência e Comportamento, Faculdade de Medicina de Ribeirão Preto, \\ Universidade de São Paulo, Ribeirão Preto, SP, Brasil \\ ${ }^{3}$ Departamento de Medicina Preventiva, Faculdade de Medicina, \\ Universidade de São Paulo, São Paulo, SP, Brasil
}

\begin{abstract}
Previous cross-sectional magnetic resonance imaging (MRI) studies of healthy aging in young adults have indicated the presence of significant inverse correlations between age and gray matter volumes, although not homogeneously across all brain regions. However, such cross-sectional studies have important limitations and there is a scarcity of detailed longitudinal MRI studies with repeated measures obtained in the same individuals in order to investigate regional gray matter changes during short periods of time in non-elderly healthy adults. In the present study, 52 healthy young adults aged 18 to 50 years (27 males and 25 females) were followed with repeated MRI acquisitions over approximately 15 months. Gray matter volumes were compared between the two times using voxel-based morphometry, with the prediction that volume changes would be detectable in the frontal lobe, temporal neocortex and hippocampus. Voxel-wise analyses showed significant $(P<0.05$, family-wise error corrected) relative volume reductions of gray matter in two small foci located in the right orbitofrontal cortex and left hippocampus. Separate comparisons for males and females showed bilateral gray matter relative reductions in the orbitofrontal cortex over time only in males. We conclude that, in non-elderly healthy adults, subtle gray matter volume alterations are detectable after short periods of time. This underscores the dynamic nature of gray matter changes in the brain during adult life, with regional volume reductions being detectable in brain regions that are relevant to cognitive and emotional processes.
\end{abstract}

Key words: Gray matter; Aging; Voxel-based morphometry; Magnetic resonance imaging; Prefrontal cortex; Hippocampus

\section{Introduction}

Studies of the processes of development and aging of the normal brain are important to increase knowledge about the physiological bases of brain functioning, and also to help clarify the pathophysiology of neurological and psychiatric disorders. In vivo research studies that use modern neuroimaging techniques such as magnetic resonance imaging (MRI) have shown several changes in the volume of the central nervous system during the life span of healthy living individuals (1-6). However, despite the large number of studies in this field, to date there is no consensus about which would be the most relevant neurobiological mechanisms in the process of cerebral aging. While some groups propose that the aging process is mainly characterized by changes in white matter associated with subcortical neuronal loss (7), others suggest that the effects of aging are primarily and substantially reflected in alterations in the volume of cortical gray matter regions $(4,8)$. Nevertheless, most researchers worldwide agree that both gray and white matter changes are important in the aging process. Moreover, there is evidence that volumetric alterations of gray matter associated with the process of brain aging occur differently according to the stage of life (childhood, adolescence, adulthood, or elderly life) (8) and also between females and males $(9,10)$.

Also, the latest publications point out that alterations in the volume of gray matter associated with aging are not

Correspondence: G.F. Busatto, Centro de Medicina Nuclear, Hospital das Clínicas, USP, Rua Dr. Ovídio Pires Campos, s/n, 2º andar, 05403-010 São Paulo, SP, Brasil. Fax: +55-11-3082-1015. E-mail: geraldo.busatto@hcnet.usp.br

Received September 25, 2011. Accepted March 19, 2012. Available online March 30, 2012. Published June 4, 2012. 
homogenous in all regions of the brain, with volume losses being much more pronounced in specific locations, mainly in neocortical regions. The most affected areas are thought to be the frontal lobe, the neocortex of the temporal lobe and the insula $(3,7,11,12)$. On the other hand, there have been findings indicative of a relative preservation of the cortical volumes in limbic and paralimbic areas until late stages of life (4). This is in accordance with the view that phylogenetically older brain areas mature earlier and take longer to undergo volumetric changes in the process of brain aging (13). However, this may not apply to all limbic brain structures; for instance, there is controversy in regard to the effects of aging on the hippocampus. While some studies show that there are only volume losses of this region in elderly life or related to pathological cerebral aging $(1,2)$, more recent investigations have shown that there is volume reduction in this area also in young healthy adults, although less marked than in the elderly $(5,14)$.

Almost all findings cited above have arisen from crosssectional MRI studies, which investigated patterns of correlation between the chronological age of healthy individuals and the corresponding gray matter volumes. Although informative, such studies provide only a superficial view of the chain of morphological brain alterations associated with aging; these investigations often examine individuals at widely variable ages during the lifespan, and rely on a single MRI data point for each of the subjects investigated.

One question that cannot be answered using these cross-sectional designs is whether there are changes in gray matter volumes in cortical regions within short periods during non-elderly adult life. This is a relevant issue, given that a number of neuropsychiatric conditions that involve anatomical and functional abnormalities of these brain regions have their onset at this stage of life. It is also of great importance to study young adults because it is possible that some of the volumetric brain changes observed in elderly individuals originated in earlier stages of adulthood. A better understanding of this normal phenomenon may help to contextualize the neurodegenerative processes associated with a number of neurological and psychiatric disorders.

The longitudinal design, in which the same individuals are repeatedly investigated with morphometric MRI over time, is potentially informative to clarify the issues cited above. During the past few years, a few longitudinal MRI studies have been published investigating morphometric brain changes over time in healthy individuals $(15,16)$. Thus, the sensitivity of MRI in detecting changes in cortical morphology during short time intervals now seems to be established. However, as is the case for the cross-sectional literature reviewed cited above, most of these longitudinal MRI studies have emphasized brain changes in elderly life $(16,17)$. These longitudinal studies have suggested that brain volume losses during short periods of time are more marked in the elderly and are not homogeneous across different brain areas (15,18-20). In general, the brain areas showing greater relative volumetric reductions are the frontal and temporal lobes, followed by the hippocampus (which displays increased volume losses in later phases of life) $(3,4)$. There are no reports of relative increases of regional brain volumes in longitudinal MRI studies of healthy aging $(19,21)$.

In the present study, we investigated brain volumetric changes over time by repeated morphometric MRI measurements in 52 healthy non-elderly adults aged 18 to 50 years. Images were acquired at two times separated by a mean period of approximately 15 months, and were processed using voxel-by-voxel analysis methods (voxelbased morphometry; VBM). We tested the hypothesis that, in non-elderly adults, there would be a modest degree of relative volume decrease (but still detectable by the VBM technique) of the gray matter during a period of 1 year, detected mainly in the frontal lobe, the neocortex of the temporal lobe and the hippocampus.

\section{Material and Methods}

\section{Study sample}

Morphometric MRI datasets of a population-based morphometric MRI study of first-episode psychosis, carried out in a circumscribed geographical area of São Paulo, Brazil (22), were used. In this baseline study, MRI data were acquired in a sample of 122 first-episode psychosis subjects, including 62 patients with schizophrenia or schizophreniform disorder, identified by active surveillance of cases that made contact for the first time with local health services for psychotic symptoms, and were compared with images of 94 age and gender-matched non-psychotic controls recruited from exactly the same geographical areas (next-door neighbors) (23). From the initial sample of 122 patients and 94 controls who participated in the baseline MRI study, 80 patients and 52 controls were rescanned after a mean time of approximately 1 year.

From the pool of healthy volunteers above, we evaluated the MRI data of 52 healthy subjects aged 18 to 50 years (27 men and 25 women). Exclusion criteria were a) history of head injury with loss of consciousness; b) presence of neurological disorders or any organic disorders that could affect the central nervous system; c) contraindications for MRI scanning; d) personal history of axis I psychiatric disorders, except mild anxiety disorders, as assessed by the Structured Clinical Interview for DSM-IV (SCID); e) current diagnosis of substance abuse or dependence diagnosis as investigated with the SCID, the Alcohol Use Disorders Identification Test (AUDIT) (24) and the South Westminster Questionnaire (25).

The study was approved by the Ethics Committee of the University Hospital, University of São Paulo, and subjects signed a consent form after being informed about the procedures involved. 


\section{Image acquisition and processing}

Imaging data were acquired using either of two identical MRI scanners (1.5 T GE Signa scanner, General Electric, USA) at the University Hospital, University of São Paulo. Exactly the same acquisition protocols were used (a T1SPGR sequence providing 124 contiguous slices, voxel size $=0.86 \times 0.86 \times 1.5 \mathrm{~mm}, \mathrm{TE}=5.2 \mathrm{~ms}, \mathrm{TR}=21.7 \mathrm{~ms}$, flip angle $=20^{\circ}, \mathrm{FOV}=22$, matrix size $=256 \times 192 \mathrm{~mm}$ ). All images were inspected visually by an experienced radiologist to identify artifacts during image acquisition and the presence of silent gross brain lesions.

The reliability of the MRI data obtained with the two scanners was assessed by intraclass correlation coefficients (ICC) calculated based on estimates of regional gray matter, obtained using the spatially normalized volumes of interest within the Automatic Anatomical Labeling ( $A A L)$ surface parametric mapping (SPM2) toolbox. Six healthy volunteers were examined twice on the same day using both scanners. We obtained ICC values higher than 0.90 for all cortical regions and medial temporal structures, and values between 0.83 (thalamus) and 0.23 (putamen) in the subcortical nuclei, as previously reported $(23,26)$.

Imaging data were processed using the Statistical Parametric Mapping package (Wellcome Department of Cognitive Neurology, Institute of Neurology, UK), executed in Matlab 6.1 version (Mathworks, USA), according to the SPM2 optimized protocol for VBM (3). This initially involved the creation of a standard template set specifically for the study, consisting of an average T1-weighted image and a priori gray matter, white matter and cerebrospinal fluid templates, based on the images of all subjects $(3,23)$. Subsequently, the original images from all subjects were processed, beginning by image segmentation with the study specific, a priori gray matter, white matter and cerebrospinal fluid templates (3). Extracted gray matter images were then spatially normalized to the customized gray matter templates with 12-parameter linear and non-linear transformations (7_9_7 basis functions). The parameters resulting from this spatial normalization step were then reapplied to the original structural images. These fully normalized images were re-sliced using tri-linear interpolation to a voxel size of $2 \times 2 \times 2 \mathrm{~mm}$ and segmented into gray matter, white matter and cerebrospinal fluid partitions. Voxel values were modulated by the Jacobian determinants derived from the spatial normalization, thus allowing brain structures that had their volumes reduced after spatial normalization to have their total counts decreased by an amount proportional to the degree of volume shrinkage (3). Finally, images were smoothed using a 12-mm Gaussian kernel to reduce variations caused by inter-individual differences in the anatomy of the gray and white matter brain compartments.

\section{Statistical analysis}

First, a comparison of the global gray matter volume between the two times was carried out in order to verify if there were overall volumetric alterations in gray matter volumes over time. Next, using the volume of global gray matter as a confounding covariate, we investigated the presence of regional foci where a greater volumetric reduction than the global loss of the gray matter would have occurred between the two times. Due to the wide inter-subject variability in regard to the interval between the two MRI acquisitions (range $=251-1159$ days, mean $=466 \pm 160$ days, median $=705$ days), the number of days between the two acquisitions was also entered in the analysis as a covariate of interest. Also, although we had obtained high ICC values in the inter-scanner reliability assessment, all statistical analyses were repeated including scanner site as a confounding covariate.

For each voxel of gray matter, paired $t$-tests were calculated comparing brain volume measurements between the first and second MRI scans. Such voxel-wise investigation was performed for the overall sample, and subsequently for the male and female subgroups separately. Only voxels with values above an absolute gray matter threshold of 0.05 entered the analyses. Resulting statistics at each voxel were transformed to Z-scores and displayed as SPMs into a standardized space, at an initial threshold of $Z>3.09$ (corresponding to one-tailed $\mathrm{P}<0.001$ level of significance, uncorrected for multiple comparisons).

In regions where volumetric changes over time had been predicted a priori (frontal lobe, temporal neocortex and hippocampus), this hypothesis-driven analysis was conducted using the small volume correction (SVC) method, with the purpose of constraining the total number of voxels included in the analyses. Each region was circumscribed using the spatially normalized region-of-interest (ROI) masks that are available within the AAL SPM toolbox. ROI masks were used for each hemisphere, involving the prefrontal cortex (dorsomedial, dorsolateral and orbitofrontal portions), lateral temporal neocortex (superior, middle and inferior temporal gyri), and temporolimbic region (amygdala, hippocampus and parahippocampal gyrus), respectively. Findings of these hypothesis-driven, SVC-based analyses were reported as significant if they survived family-wise error (FWE) correction for multiple comparisons $(P<0.05)$ over the respective volume of interest. In all analyses, we converted MNI coordinates of voxels of maximal statistical significance to the Talairach and Tournoux coordinates (27).

\section{Results}

The sum of intensity values of all voxels in the brain obtained from the segmented gray matter images had a mean value of $71,384.67(\mathrm{SD}=7479.09)$ in the first MRI scans, and 71,634.71 $(\mathrm{SD}=7781.08)$ in the second MRI scans $(t=-1.03$, d.f. $=51, P=0.306)$. This indicated no overall volumetric alterations over time.

The SVC-based analyses comparing regional brain vol- 
umes between the two MRI data points are reported in Table 1 , with covariance for inter-scanning interval. This analysis demonstrated the presence of two small foci of significant relative decrease of gray matter in some portions (P-FWE $\leq 0.05$ ) of the brain structures where significant findings had been hypothesized a priori (Table 1), namely the right orbitofrontal portion of the frontal lobe (Figure 1A) and the left hippocampus (Figure 1B). No areas of relative volume increase over time were found. Also, contrary to the initial prediction of the study, no volume changes were observed in the neocortex of the temporal lobe (Table 1).

The same overall pattern of results was obtained when the above VBM analyses were repeated including scanner site (1 or 2) as a nuisance covariate (Table 2), with relative gray matter reductions being detected again in the frontal lobe (right orbital frontal cortex) and left hippocampus.

When comparisons of brain volumes between the two MRI data points were carried out separately for females and males, the finding of a relative decrease in orbitofrontal gray matter retained significance in the male subgroup both for the right and left hemisphere, while the hippocampus showed no alterations in its gray matter volume over time either in males or females (Table 3).

Finally, due to the relatively large age range of the present sample, we conducted separate longitudinal analyses for the subgroups of individuals aged 18 to 30 years $(N=29)$,

Table 1. Hypothesis-driven search for significant gray matter volume changes during approximately 15 months in healthy individuals $(\mathrm{N}=52)$.

\begin{tabular}{|c|c|c|c|c|c|c|c|c|}
\hline \multirow[t]{2}{*}{ Brain regions $(\mathrm{SVC})^{\mathrm{a}}$} & \multirow[t]{2}{*}{$\begin{array}{l}\text { Direction of } \\
\text { changes }^{b}\end{array}$} & \multirow[t]{2}{*}{ Hemisphere } & \multirow[t]{2}{*}{ Peak Z-scores ${ }^{c}$} & \multicolumn{3}{|c|}{$\begin{array}{l}\text { Talairach and Tournoux } \\
\text { coordinates (peak voxels) }\end{array}$} & \multirow[t]{2}{*}{$\begin{array}{l}\text { Number } \\
\text { of voxels }\end{array}$} & \multirow[t]{2}{*}{$P[F W E]^{d}$} \\
\hline & & & & $x$ & $\mathrm{y}$ & z & & \\
\hline \multirow[t]{2}{*}{ Frontal cortex } & Negative & Right & 3.68 (orbitofrontal) & 30 & 42 & -16 & 259 & 0.009 \\
\hline & & Left & - & - & - & - & - & - \\
\hline Temporal cortex & - & - & - & - & - & - & - & - \\
\hline \multirow[t]{2}{*}{ Hippocampus } & Negative & Right & - & - & - & - & - & - \\
\hline & & Left & 3.13 & -32 & -38 & 0 & 97 & 0.049 \\
\hline
\end{tabular}

aEach region was circumscribed using the small volume correction (SVC) approach, with anatomically defined volume-of-interest

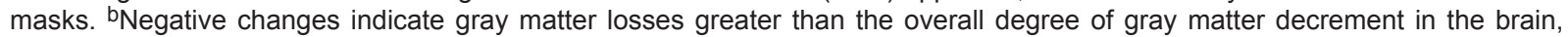
including the time interval between the two scans as a covariate of interest. 'Z-score for the voxel of peak statistical significance within each volume of interest (name of the corresponding anatomical brain structure in parentheses). dFamily-wise error (FWE) correction for multiple comparisons at the level of individual voxels within the respective volume of interest.
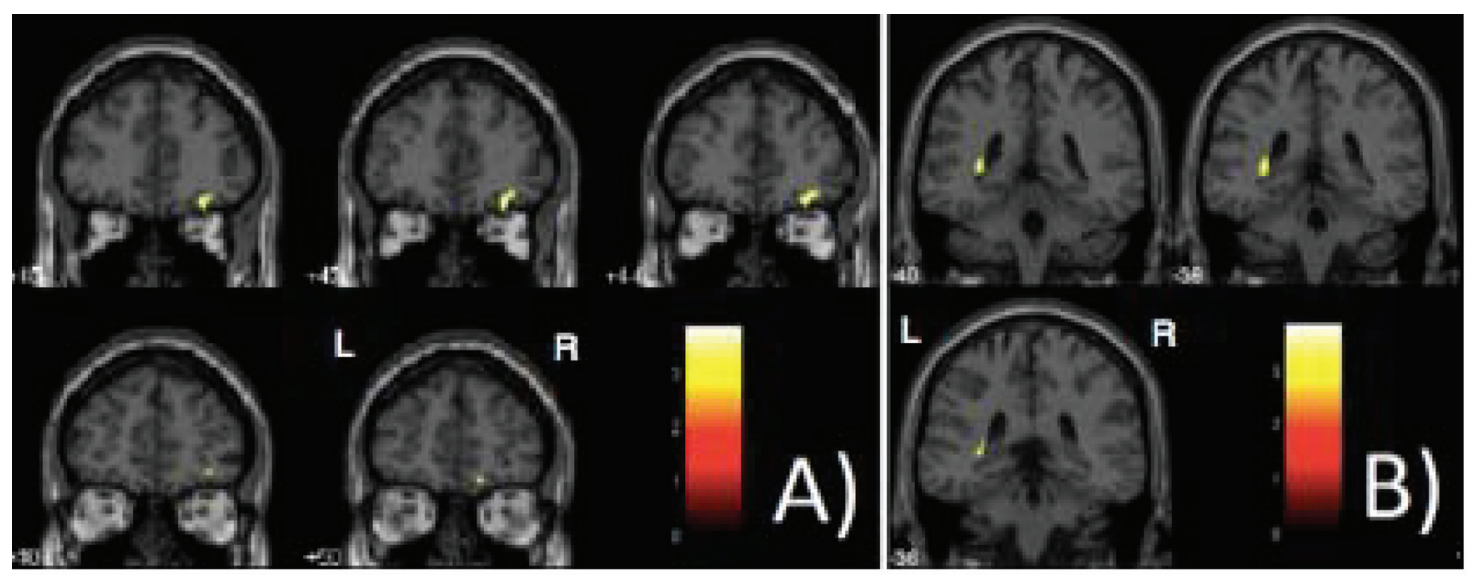

Figure 1. Clusters of significant relative decrease in the gray matter volume obtained from voxel-based morphometry analyses (paired $t$-tests were calculated comparing brain volume measurements between the first and second MRI scans, $\mathrm{P}<0.05$, family-wise error-corrected for multiple comparisons). The right orbitofrontal portion of the frontal lobe $(A)$ and the left hippocampus $(B)$ are highlighted in yellow, overlaid on coronal slices spatially normalized into an approximation to the Talairach and Tournoux (27) stereotactic atlas. The analysis was controlled for differences between subjects in regard to the time interval between the two MRI acquisitions, entered as a covariate of interest. $L=$ left; $R=$ right. 
Table 2. Hypothesis-driven search for significant gray matter volume changes including scanner site as confounding covariate during approximately 15 months in healthy individuals $(N=52)$.

\begin{tabular}{|c|c|c|c|c|c|c|c|c|}
\hline \multirow[t]{2}{*}{ Brain regions $(\mathrm{SVC})^{\mathrm{a}}$} & \multirow[t]{2}{*}{$\begin{array}{l}\text { Direction of } \\
\text { changes }^{b}\end{array}$} & \multirow[t]{2}{*}{ Hemisphere } & \multirow[t]{2}{*}{ Peak Z-scores ${ }^{c}$} & \multicolumn{3}{|c|}{$\begin{array}{l}\text { Talairach and Tournoux } \\
\text { coordinates (peak voxels) }\end{array}$} & \multirow[t]{2}{*}{$\begin{array}{l}\text { Number } \\
\text { of voxels }\end{array}$} & \multirow[t]{2}{*}{$P[F W E]^{d}$} \\
\hline & & & & $x$ & $y$ & z & & \\
\hline \multirow[t]{2}{*}{ Frontal cortex } & \multirow[t]{2}{*}{ Negative } & Right & 3.70 (orbitofrontal) & 30 & 42 & -16 & 223 & 0.009 \\
\hline & & Left & - & - & - & - & - & - \\
\hline Temporal cortex & - & - & - & - & - & - & - & - \\
\hline \multirow[t]{2}{*}{ Hippocampus } & Negative & Right & - & - & - & - & - & - \\
\hline & & Left & 3.11 & -30 & -38 & 2 & 98 & 0.005 \\
\hline
\end{tabular}

${ }^{a}$ Each region was circumscribed using the small volume correction (SVC) approach, with anatomically defined volume-of-interest masks. bNegative changes indicate gray matter losses greater than the overall degree of gray matter decrement in the brain, including the time interval between the two scans as a covariate of interest. CZ-score for the voxel of peak statistical significance within each volume of interest (name of the corresponding anatomical brain structure in parentheses). ${ }^{\mathrm{d}}$ Family-wise error (FWE) correction for multiple comparisons at the level of individual voxels within the respective volume of interest.

Table 3. Hypothesis-driven search for significant gray matter volume changes during approximately 15 months in healthy men $(\mathrm{N}=27)$.

\begin{tabular}{|c|c|c|c|c|c|c|c|c|}
\hline \multirow[t]{2}{*}{ Brain regions $(S V C)^{a}$} & \multirow[t]{2}{*}{$\begin{array}{l}\text { Direction of } \\
\text { changes }^{b}\end{array}$} & \multirow[t]{2}{*}{ Hemisphere } & \multirow[t]{2}{*}{ Peak Z-scores ${ }^{c}$} & \multicolumn{3}{|c|}{$\begin{array}{l}\text { Talairach and Tournoux } \\
\text { coordinates (peak voxels) }\end{array}$} & \multirow[t]{2}{*}{$\begin{array}{l}\text { Number } \\
\text { of voxels }\end{array}$} & \multirow[t]{2}{*}{$P[F W E]^{d}$} \\
\hline & & & & $x$ & $y$ & z & & \\
\hline \multirow[t]{2}{*}{ Frontal cortex } & Negative & Right & 3.29 (orbitofrontal) & 16 & 46 & -16 & 109 & 0.037 \\
\hline & & Left & 3.46 (orbitofrontal) & -16 & 44 & -16 & 116 & 0.021 \\
\hline Temporal cortex & - & - & - & - & - & - & - & - \\
\hline Hippocampus & - & - & - & - & - & - & - & - \\
\hline
\end{tabular}

aEach region was circumscribed using the small volume correction (SVC) approach, with anatomically defined volume-of-interest masks. bNegative changes indicate gray matter losses greater than the overall degree of gray matter decrement in the brain, including the time interval between the two scans as a covariate of interest. CZ-score for the voxel of peak statistical significance within each volume of interest (name of the corresponding anatomical brain structure in parentheses). dFamily-wise error (FWE) correction for multiple comparisons at the level of individual voxels within the respective volume of interest.

and for the subgroup older than 30 years $(N=23)$. These analyses showed no brain areas of relative volume decrease or increase in either of these two subgroups over time.

\section{Discussion}

This longitudinal MRI study investigated the presence of gray matter volume loss over time in a sample of non-elderly healthy adults, with morphometric brain measurements separated by an average of approximately 15 months. Based on previous MRI studies of healthy aging, we selected three brain regions for our search for gray matter changes, and identified small foci of volume reductions in two of them, namely the prefrontal cortex and hippocampus. Although previous longitudinal MRI studies of healthy aging in non- elderly adults have reported negative results, several other such longitudinal investigations are consistent with the findings of the present study $(15-17,19,21)$. Such previous longitudinal MRI studies have indicated that the frontal and temporal lobes are the sites of greater gray matter reductions over time in non-elderly adulthood $(16,19)$, followed by the hippocampal region $(15,21)$. Thus, in non-elderly healthy adults, subtle gray matter volume decrements are detectable even after short periods of time. This underscores the dynamic nature of gray matter changes in the brain during adult life, with regional volume reductions being detectable in brain regions that are relevant to cognitive and emotional processes over periods as short as approximately 1 year.

Rather than the dorsolateral prefrontal cortex, the orbitofrontal cortex was the prefrontal subregion in which 
significant gray matter relative volume reductions were detected in our sample of non-elderly adults. This is not entirely consistent with the findings of previous crosssectional MRI investigations (2), including our own study, which was based on the baseline MRI measurements of the healthy population from which the current sample was drawn (26). Given that phylogenetically more recent brain regions would be expected to be the first to suffer with the process of brain aging (13), one would expect greater gray matter reductions in dorsolateral prefrontal regions than in the orbitofrontal cortex in association with non-elderly adult aging. It is possible that significant gray matter losses in the dorsolateral prefrontal cortex are either absent over a short period of adult life such as a single year or too subtle to be detectable with VBM methods such as those used herein.

One interesting aspect of our study concerns the differences in aging-related gray matter changes between genders, with frontal cortical volume decrement being detected in males but not in females. Gender-related differences have been demonstrated in a number of cross-sectional $(3,28)$ and longitudinal $(16,17)$ MRI studies of healthy aging, involving the frontal cortex and other brain regions. Among the factors that could possibly explain the differential regional brain vulnerability to normal aging between males and females, the influence of sex hormones has raised particular interest $(29,30)$. Several studies have indicated that sex hormones, most specifically the female estrogens, may play a role in the maintenance of neocortical structural and functional integrity over adult life (31-33).

Our results regarding the hippocampus are consistent with the findings of previous longitudinal MRI investigations of healthy subjects followed up over longer periods of adult life (19,30-32). These results add evidence refuting the notion that gray matter reductions in this brain region occur only in elderly life or in association with pathological neurodegenerative processes, such as those that characterize Alzheimer's disease $(3,4,34)$. Thus, our results, added to previous literature findings, reinforce the notion that the hippocampus is not spared in normal aging, indicating that this brain structure may undergo significant reductions in gray matter volume even within short periods of non-elderly life (35).

The above hippocampal findings from a within-group longitudinal comparison contrast with the results of a number of cross-sectional MRI studies, which have reported no direct correlations between age and hippocampal gray matter volumes in healthy adults $(5,14)$. Indeed, in our own cross-sectional investigation of age-related gray matter changes (26), there were inverse rather than direct correlations between hippocampal gray matter volumes and age, thus suggesting volume preservation of this brain region during non-elderly adult life (26). Such distinct findings indicate that different information can be obtained in MRI studies of healthy aging when either cross-sectional or longitudinal experimental designs are used. This highlights the importance of complementing these two experimental views with each other, in order to afford a greater understanding of the processes that characterize the effects of aging on healthy brains.

The age range of the subjects included in the current study (18 to 50 years) was relatively wide. Therefore, it is difficult to ascertain whether the gray matter decline detected herein is predominantly related to maturational or agerelated degenerative processes, which are likely to occur at different degrees across separate stages of life within this wide age range. The brain volumetric changes reported herein probably reflect loss of dendrites and shrinkage of neurons (36). Maturational, synaptic pruning changes may contribute to the thinning of the cerebral cortex in subjects of younger age (12).

Age-related microstructural changes over time, whether maturational or degenerative, are also likely to differ across distinct regions of the brain in different stages of adult life. We attempted to deal with the problem of the wide age range of our sample by conducting separate analyses for the subgroup of individuals aged 18 to 30 years and for the subgroup older than 30 years. However, such separate analyses revealed no areas of relative increase or decrease of gray matter in either subgroup. Such negative results are likely to be due to insufficient power of our study, given the relatively modest number of subjects in each of those two age subgroups. Further MRI studies with larger samples are needed in order to verify whether the regional gray matter decline reported herein is present uniformly over the three decades spanned by our study, or varies both in degree and regional specificity across different stages of non-elderly adult life.

An additional aspect of our study that deserves mentioning is the lateralization of the main results (right orbitofrontal cortex and left hippocampus). Lateralized findings have often been reported in previous MRI studies of healthy aging $(4,10)$. The human brain is known to be asymmetric, and between-hemisphere gray matter differences are thought to be relevant to support higher-order cognitive functions (37). Findings of lateralized gray matter decrements over time may be relevant to the subtle cognitive decline often associated with healthy aging in humans.

\section{Limitations}

The results of the current study should be interpreted with caution due to its several limitations. These include the wide age range of the subjects investigated as well as the variable interval between the two MRI acquisitions. We have controlled our comparisons for such influences, but the high variability of these variables may have obscured the detection of further significant findings. It is therefore plausible that other foci of gray matter reductions from the first to the second MRI acquisition might have been detected in a sample with a more homogeneous age range and less 
variable interscan intervals. Another important limitation of our study is that we combined imaging data acquired using two different MRI scanners. However, the two scanners and acquisition protocols were identical, and we obtained very high inter-equipment reliability indices for the neocortical and limbic regions that were the main focus of the investigation. Additionally, the inclusion of scanner site as a covariate did not alter the results of the VBM analyses. It should also be noted that even when a single MRI scanner is used in longitudinal studies, issues of gradient non-linearity and other distortions may influence the detection of any real structural brain changes occurring within short periods of time in the healthy population.

Limitations of the VBM methodology should also be highlighted, including the risk of systematic registration errors during spatial normalization (38) and segmentation biases in brain areas where tissue contrast is poorly defined in MRI scans (39). Moreover, it should be noted that newer versions of the SPM package than the one employed herein have been released in the past few years (SPM5 and SPM8). Although we have incorporated the latest SPM versions in recent VBM studies by our group, we believe that caution should be exercised when considering their use for longitudinal VBM investigations of aging-related gray matter changes, as attempts to validate these novel SPM versions for longitudinal VBM studies have only very recently started to appear in the literature. Finally, although

\section{References}

1. Abe O, Yamasue $\mathrm{H}$, Aoki $\mathrm{S}$, Suga M, Yamada H, Kasai $\mathrm{K}$, et al. Aging in the CNS: comparison of gray/white matter volume and diffusion tensor data. Neurobiol Aging 2008; 29 : 102-116.

2. Alexander GE, Chen K, Merkley TL, Reiman EM, Caselli $\mathrm{RJ}$, Aschenbrenner M, et al. Regional network of magnetic resonance imaging gray matter volume in healthy aging. Neuroreport 2006; 17: 951-956.

3. Good CD, Johnsrude IS, Ashburner J, Henson RN, Friston KJ, Frackowiak RS. A voxel-based morphometric study of ageing in 465 normal adult human brains. Neuroimage 2001; 14: 21-36.

4. Grieve SM, Clark CR, Williams LM, Peduto AJ, Gordon E. Preservation of limbic and paralimbic structures in aging. Hum Brain Mapp 2005; 25: 391-401.

5. Kalpouzos G, Chetelat G, Baron JC, Landeau B, Mevel K, Godeau $\mathrm{C}$, et al. Voxel-based mapping of brain gray matter volume and glucose metabolism profiles in normal aging. Neurobiol Aging 2009; 30: 112-124.

6. Taki Y, Goto R, Evans A, Zijdenbos A, Neelin P, Lerch J, et al. Voxel-based morphometry of human brain with age and cerebrovascular risk factors. Neurobiol Aging 2004; 25: 455463.

7. Jernigan TL, Archibald SL, Fennema-Notestine C, Gamst AC, Stout JC, Bonner J, et al. Effects of age on tissues and regions of the cerebrum and cerebellum. Neurobiol Aging 2001; 22: 581-594. the current VBM protocol has been often employed in longitudinal MRI studies of neuropsychiatric disorders, it should be acknowledged that its use of low frequency basis functions during spatial normalization may reduce sensitivity in detecting subtle gray matter differences as expected in longitudinal investigations. Methods that afford such gain in sensitivity for longitudinal MRI studies include the techniques of deformation-based morphometry and tensor-based morphometry. Therefore, replication of the current findings with such methods is warranted.

\section{Acknowledgments}

We thank Claudia C. Leite, Edson Amaro Jr., and Claudio C. de Castro for assistance with the acquisition of MRI data, and for the visual inspection of images to detect gross brain abnormalities. This study was carried out using clinical and MRI datasets acquired for a previous research project supported by FAPESP (\#08/53006-8), and the Wellcome Trust, UK (\#GR066133MA). We acknowledge the contribution of the researchers of the Brazilian First Contact Psychosis team (funded by the Wellcome Trust) to the demographic and clinical evaluation of the healthy volunteers selected for the current study, as well as the grant co-holders Robin M. Murray and Philip K. McGuire. M. Scazufca, P.R. Menezes, and G.F. Busatto are supported by CNPq.

8. Sowell ER, Thompson PM, Toga AW. Mapping changes in the human cortex throughout the span of life. Neuroscientist 2004; 10: 372-392.

9. Chen X, Sachdev PS, Wen W, Anstey KJ. Sex differences in regional gray matter in healthy individuals aged 44-48 years: a voxel-based morphometric study. Neuroimage 2007; 36 : 691-699.

10. Curiati PK, Tamashiro JH, Squarzoni P, Duran FL, Santos $\mathrm{LC}$, Wajngarten $\mathrm{M}$, et al. Brain structural variability due to aging and gender in cognitively healthy elders: results from the São Paulo Ageing and Health study. AJNR Am J Neuroradiol 2009; 30: 1850-1856.

11. Salat DH, Kaye JA, Janowsky JS. Prefrontal gray and white matter volumes in healthy aging and Alzheimer disease. Arch Neurol 1999; 56: 338-344.

12. Sowell ER, Thompson PM, Tessner KD, Toga AW. Mapping continued brain growth and gray matter density reduction in dorsal frontal cortex: Inverse relationships during postadolescent brain maturation. J Neurosci 2001; 21: 8819-8829.

13. Toga AW, Thompson PM, Mori S, Amunts K, Zilles K. Towards multimodal atlases of the human brain. Nat Rev Neurosci 2006; 7: 952-966.

14. Xu Y, Valentino DJ, Scher AI, Dinov I, White LR, Thompson $\mathrm{PM}$, et al. Age effects on hippocampal structural changes in old men: the HAAS. Neuroimage 2008; 40: 1003-1015.

15. Raz N, Ghisletta P, Rodrigue KM, Kennedy KM, Lindenberger $\mathrm{U}$. Trajectories of brain aging in middle-aged and 
older adults: regional and individual differences. Neuroimage 2010; 51: 501-511.

16. Thambisetty M, Wan J, Carass A, An Y, Prince JL, Resnick SM. Longitudinal changes in cortical thickness associated with normal aging. Neuroimage 2010; 52: 1215-1223.

17. Driscoll I, Davatzikos C, An Y, Wu X, Shen D, Kraut M, et al. Longitudinal pattern of regional brain volume change differentiates normal aging from MCl. Neurology 2009; 72 : 1906-1913.

18. Fjell AM, Walhovd KB, Fennema-Notestine C, McEvoy LK, Hagler DJ, Holland D, et al. One-year brain atrophy evident in healthy aging. $J$ Neurosci 2009; 29: 15223-15231.

19. Raz N, Lindenberger U, Rodrigue KM, Kennedy KM, Head $\mathrm{D}$, Williamson $\mathrm{A}$, et al. Regional brain changes in aging healthy adults: general trends, individual differences and modifiers. Cereb Cortex 2005; 15: 1676-1689.

20. Resnick SM, Pham DL, Kraut MA, Zonderman AB, Davatzikos $C$. Longitudinal magnetic resonance imaging studies of older adults: a shrinking brain. $J$ Neurosci 2003; 23: 3295-3301.

21. Liu RS, Lemieux L, Bell GS, Sisodiya SM, Shorvon SD, Sander JW, et al. A longitudinal study of brain morphometrics using quantitative magnetic resonance imaging and difference image analysis. Neuroimage 2003; 20: 22-33.

22. Menezes PR, Scazufca M, Busatto G, Coutinho LM, McGuire PK, Murray RM. Incidence of first-contact psychosis in São Paulo, Brazil. Br J Psychiatry Suppl 2007; 51: s102-s106.

23. Schaufelberger MS, Duran FL, Lappin JM, Scazufca M, Amaro E Jr, Leite CC, et al. Grey matter abnormalities in Brazilians with first-episode psychosis. Br J Psychiatry Suppl 2007; 51: s117-s122.

24. Saunders JB, Aasland OG, Babor TF, de la Fuente Jr, Grant M. Development of the Alcohol Use Disorders Identification Test (AUDIT): WHO Collaborative Project on Early Detection of Persons with Harmful Alcohol Consumption - II. Addiction 1993; 88: 791-804.

25. Menezes PR, Johnson S, Thornicroft G, Marshall J, Prosser $D$, Bebbington $P$, et al. Drug and alcohol problems among individuals with severe mental illness in south London. $\mathrm{Br} J$ Psychiatry 1996; 168: 612-619.

26. Terribilli D, Schaufelberger MS, Duran FL, Zanetti MV, Curiati PK, Menezes PR, et al. Age-related gray matter volume changes in the brain during non-elderly adulthood. Neurobiol Aging 2011; 32: 354-368.

27. Talairach J, Tournoux P. Co-planar stereotactic atlas of the human brain. Stuttgart/New York: Thieme; 1988.
28. Greenberg DL, Messer DF, Payne ME, MacFall JR, Provenzale JM, Steffens DC, et al. Aging, gender, and the elderly adult brain: an examination of analytical strategies. Neurobiol Aging 2008; 29: 290-302.

29. Berman KF, Schmidt PJ, Rubinow DR, Danaceau MA, Van Horn JD, Esposito G, et al. Modulation of cognition-specific cortical activity by gonadal steroids: a positron-emission tomography study in women. Proc Natl Acad Sci U S A 1997; 94: 8836-8841.

30. Eberling JL, Wu C, Tong-Turnbeaugh R, Jagust WJ. Estrogen- and tamoxifen-associated effects on brain structure and function. Neuroimage 2004; 21 : 364-371.

31. Cowell PE, Sluming VA, Wilkinson ID, Cezayirli E, Romanowski CA, Webb JA, et al. Effects of sex and age on regional prefrontal brain volume in two human cohorts. Eur J Neurosci 2007; 25: 307-318.

32. Fernandez G, Weis S, Stoffel-Wagner B, Tendolkar I, Reuber $M$, Beyenburg $S$, et al. Menstrual cycle-dependent neural plasticity in the adult human brain is hormone, task, and region specific. J Neurosci 2003; 23: 3790-3795.

33. Keenan PA, Ezzat WH, Ginsburg K, Moore GJ. Prefrontal cortex as the site of estrogen's effect on cognition. Psychoneuroendocrinology 2001; 26: 577-590.

34. Raji CA, Lopez OL, Kuller LH, Carmichael OT, Becker JT. Age, Alzheimer disease, and brain structure. Neurology 2009; 73: 1899-1905.

35. Walhovd KB, Westlye LT, Amlien I, Espeseth T, Reinvang I, Raz N, et al. Consistent neuroanatomical age-related volume differences across multiple samples. Neurobiol Aging 2011; 32: 916-932.

36. Fjell AM, Walhovd KB. Structural brain changes in aging: courses, causes and cognitive consequences. Rev Neurosci 2010; 21: 187-221.

37. Watkins KE, Paus T, Lerch JP, Zijdenbos A, Collins DL, Neelin $P$, et al. Structural asymmetries in the human brain: a voxel-based statistical analysis of $142 \mathrm{MRI}$ scans. Cereb Cortex 2001; 11: 868-877.

38. Bookstein FL. "Voxel-based morphometry" should not be used with imperfectly registered images. Neuroimage 2001; 14: 1454-1462.

39. Kennedy KM, Erickson KI, Rodrigue KM, Voss MW, Colcombe SJ, Kramer AF, et al. Age-related differences in regional brain volumes: a comparison of optimized voxelbased morphometry to manual volumetry. Neurobiol Aging 2009; 30: 1657-1676. 